\title{
Erratum
}

\section{Erratum: Sartor et al., "Enhancement of BDNF Expression and Memory by HDAC Inhibition Requires BET Bromodomain Reader Proteins"}

In the article "Enhancement of BDNF Expression and Memory by HDAC Inhibition Requires BET Bromodomain Reader Proteins" by Gregory C. Sartor, Andrea M. Malvezzi, Ashok Kumar, Nadja S. Andrade, Hannah J. Wiedner, Samantha J. Vilca, Karolina J. Janczura, Amir Bagheri, Hassan Al-Ali, Samuel K. Powell, Peyton T. Brown, Claude H. Volmar, Thomas C. Foster, Zane Zeier, and Claes Wahlestedt, which published online on January 23, 2019, an author's name appears incorrectly. Samantha J. Vilca should be Samara J. Vilca. This correction does not affect the conclusions of the paper. The name has been corrected in the online record.

DOI: 10.1523/JNEUROSCI.2974-19.2019 